\title{
Shape Anisotropy of Electromagnetic Materials in Application of Microwave by Micro-sized Numerical Electromagnetic Field Calculation
}

\author{
Keisuke FUJISAKI \\ Toyota Technological Institute, 2-12-1 Hisakata, Tempaku, Nagoya, 468-8511 Japan. E-mail: fujisaki@toyota-ti.ac.jp
}

(Received on July 25, 2011; accepted on October 25, 2011)

\begin{abstract}
As the fundamental characteristics of microwave application, which is expected to be one of the useful technologies in the reduction reaction of iron oxide, electromagnetic anisotropic characteristics of electromagnetic materials in application of microwave are observed by micro-sized numerical high frequency electromagnetic computation. In assuming that the micro-sized unit structure is repeated in 3-dimensional directions and its size is much smaller than the electromagnetic wave length, the micro-sized electromagnetic field calculation using finite element method and $\mathrm{j} \omega$ method is carried out. In considering the bar shape and the cube shape as a micro-structure of electromagnetic material, the bar shape micro-structure has anisotropic characteristics, though cube shape micro-structure has isotropic ones. The generation of depolarization field within the micro-structure causes the difference of electromagnetic characteristics and the anisotropy.
\end{abstract}

KEY WORDS: microwave application; shape anisotropy; electromagnetic field; electromagnetic constant; bar shape; micro-structure; micro-sized model.

\section{Introduction}

Microwave application to electromagnetic material often introduces abnormal phenomena such as raising the boiling temperature, ${ }^{1)}$ changing the conditions of chemical reaction, ${ }^{2)}$ promoting the nitriding reaction ${ }^{3)}$ and the reduction reaction. ${ }^{4}$ Especially the reduction reaction of iron oxide in the microwave application is expected to be one of the useful technologies to solve the $\mathrm{CO}_{2}$ problem. ${ }^{5)}$ Though the reduction reaction used by carbon theoretically causes the $\mathrm{CO}_{2}$ problem, the electrical energy application, which has a lot of energy source such as solar energy or wind energy, is fundamentally possible to avoid the $\mathrm{CO}_{2}$ generation.

Moreover, microwave application to steel making plant is already on-line used or researched to obtain the superior process characteristics. A microwave dry process of monolithic refractories is the first one which has been on-line used since 1979. ${ }^{6}$ Crystallization of blast furnace slag is also researched. ${ }^{7)}$

However, though a lot of experimental data are shown to clarify the reduction reaction and to obtain the useful suggestions, ${ }^{8,9)}$ some of them are not explained theoretically enough. Numerical calculation in combination with the experimental method is expected to be an important one to solve it and to obtain the improved process.

These microwave application phenomena are considered to be caused by current generation in dielectric material by electrical field or in electrical conductive material by magnetic field. ${ }^{10,11)}$ Since the current generates within the microstructure, which is much smaller than the electromagnetic wave length, it plays an important role to decide the phenomena through the electromagnetic characteristics in the micro-structure.

Since it is difficult to measure the electromagnetic field distribution in micro-sized structure, a numerical calculation model is researched. It is clarified a detailed phenomenon of micro-sized electromagnetic field. ${ }^{12,13)}$ Then the micro-sized model is a useful method to evaluate the electromagnetic characteristics of micro-size structure material in microwave application.

Usually electromagnetic field distribution within microstructure is considered to depend on the shape, the direction and the size of the electromagnetic material. In iron oxide application, its structure is considered to have a lot of kinds of shape and size. ${ }^{14)}$ Water shape in the monolithic refractories in microwave application is also considered to be the same.

When the micro-structure is not symmetry in 3-dimensional space, electromagnetic characteristics are different in applied direction of high frequency electromagnetic field. Then the shape anisotropy of electromagnetic field is considered to be observed. In experimental data of the microwave application to iron oxide, the measured permeability is not proportion to the volume rate. One of the reasons is considered to be that its shape affected the electromagnetic characteristics. $^{15)}$

In this paper, shape anisotropy of micro-structure is researched as the fundamental electromagnetic characteristics of microwave application to electromagnetic materials, as well as iron oxide. Micro-sized water bar, to which high 
frequency electromagnetic field is applied, is considered as an asymmetry micro-structure material here.

\section{Micro-sized Electromagnetic Model}

\subsection{Micro-sized Numerical Model}

The micro-sized structure is considered to be much smaller than the wave length of high frequency electromagnetic field as shown in Fig. 1. Cube shape, which is $0.8 \times 0.8 \times$ $0.8 \mu \mathrm{m}^{3}$, and bar shape, which has $0.7155 \times 0.7155 \mu \mathrm{m}^{2}$ cross section, are considered as a micro-structure. Wave length of $125 \mathrm{~mm}(2.4 \mathrm{GHz})$ is considered as high frequency electromagnetic field. The frequency of $2.4 \mathrm{GHz}$ is selected, because it is almost the same as the most popular frequency in home apparatus as $2.45 \mathrm{GHz}$. The calculated basic size of about $1 \mu \mathrm{m}$ is selected, because it is a medium size of iron oxide powder (submicron size) ${ }^{14)}$ and $\mathrm{Fe}_{2} \mathrm{O}_{3}$ powder (38-62 $\mu \mathrm{m}){ }^{15)}$

Because of a micro-sized problem, normal propagation theory of electromagnetic wave is much difficult to be applied to it. To solve it, a micro-sized numerical model is considered, where two suppositions are assumed: 1. Wave length is much larger than micro-structure, 2. Micro-structure has a basic unit which repeats endlessly toward 3-directional space. In the case of the cube shape in Fig. $1,1 \mu \mathrm{m}^{3}$-cube is

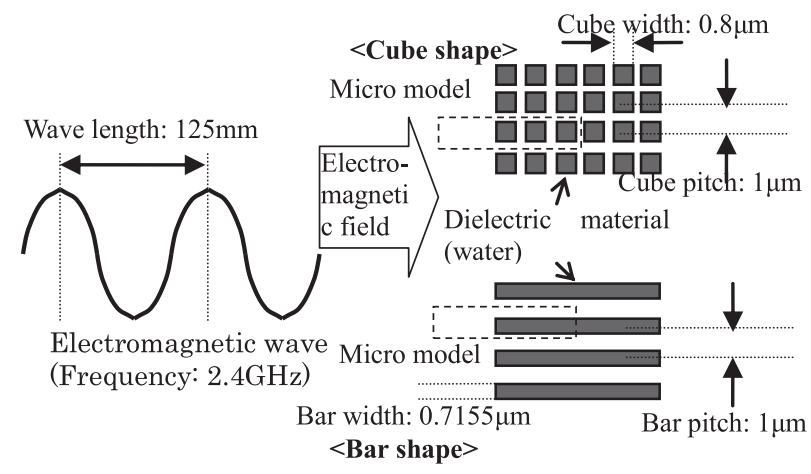

Fig. 1. Micro-sized dielectric materials and macro-sized electromagnetic wave.

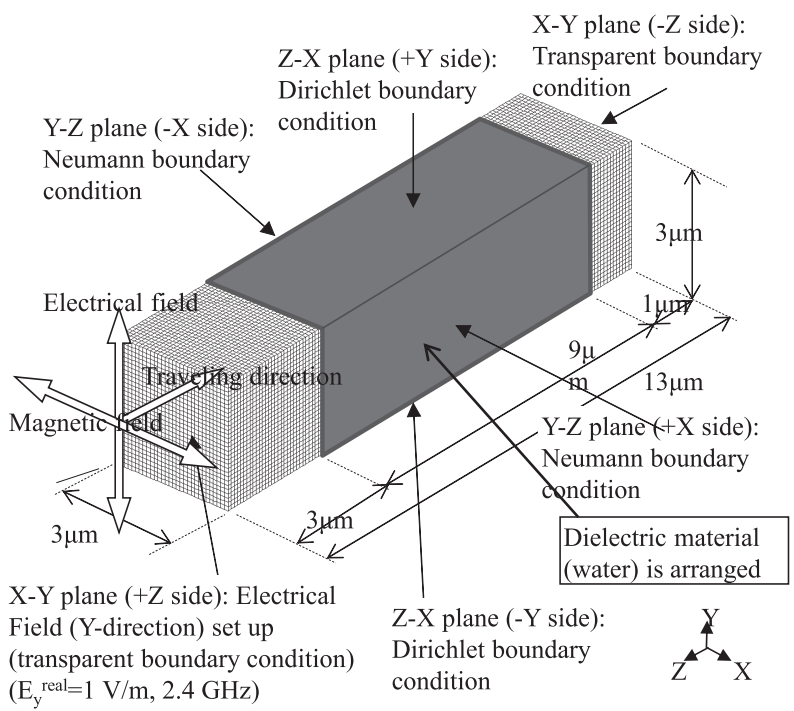

Fig. 2. Micro-sized numerical model and boundary conditions. a basic unit.

By considering the two assumptions, the boundary conditions are shown as Fig. 2. The micro-structure to be calculated is arranged in the center gray part as $3 \mu \mathrm{m} \times 3 \mu \mathrm{m} \times$ $9 \mu \mathrm{m}$ in Fig. 2. Details are shown in later.

\subsection{Electromagnetic Field Model}

All the items including eddy current and displacement current in Maxwell's equations are considered. Transient analysis method such as FDTD (Finite Difference Time Domain method) is impossible to be applied to the micromodel practically, because it takes a lot of time to pass through the micro-model. Then finite element method of $j \omega$ method is used here because it is a quisistationary state model. Then the equation using A- $\varphi$ method is as follows.

$$
\nabla \times\left([\dot{\mu}]^{-1} \nabla \times \dot{\vec{A}}\right)+([\sigma]+j \omega[\dot{\varepsilon}])(j \omega \dot{\vec{A}}+\nabla \dot{\phi})=0 \ldots
$$

Here, $\dot{\vec{A}}$ is vector potential, $\dot{\phi}$ is scholar potential, $[\dot{\mu}]$ is magnetic permeability, $[\sigma]$ is electrical conductivity, $[\dot{\varepsilon}]$ is dielectric constant. Dot on the letter expresses complex number.

\subsection{Bar Shape Model}

As a micro-structure to be evaluated, bar shape one is considered here as Fig. 3. The bar shape, which is $0.7155 \times$ $0.7155 \mu \mathrm{m}^{2}$ cross section, extends to the $\mathrm{z}$-direction endlessly and is arranged in $1 \mu \mathrm{m}$ bar pitch to $\mathrm{x}$ - and $\mathrm{y}$-directions endlessly.

Electromagnetic field is considered to be applied to it in 3-directions as $\mathrm{x}-$, $\mathrm{y}$ - and $\mathrm{z}$-directions. The one with applied direction (traveling direction of electromagnetic field) as $x-$, $\mathrm{y}$ - and $\mathrm{z}$-directions is considered to have an electrical field as $y-, \mathrm{z}$ - and $\mathrm{x}$-directions respectively. Therefore, the microsized numerical models, which are arranged at the center part in Fig. 2, are Figs. 4(a), 4(b), 4(c). The electrical field of $\mathrm{y}-, \mathrm{z}-$ and $\mathrm{x}$-directions corresponds to case-A, B and C respectively.

In all these cases, the same object of bar structure is applied to, but the directions of applying electromagnetic field are different.

The case $\mathrm{A}-\mathrm{C}$ of bar structure has the same volume rate of water as $51.2 \%$. Then the cube structure with the same volume rate is considered as Fig. 4(d) (Case-D) for compar-

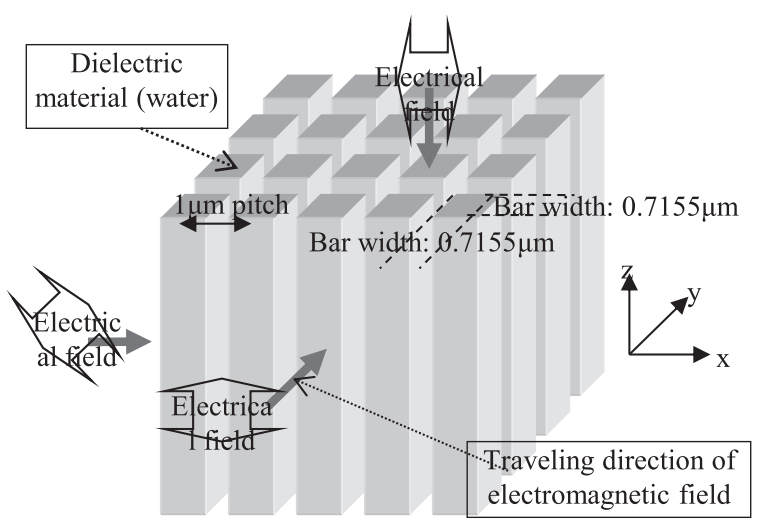

Fig. 3. Bar shape as a micro-structure to be evaluated here. Dielectric material as water is considered. 


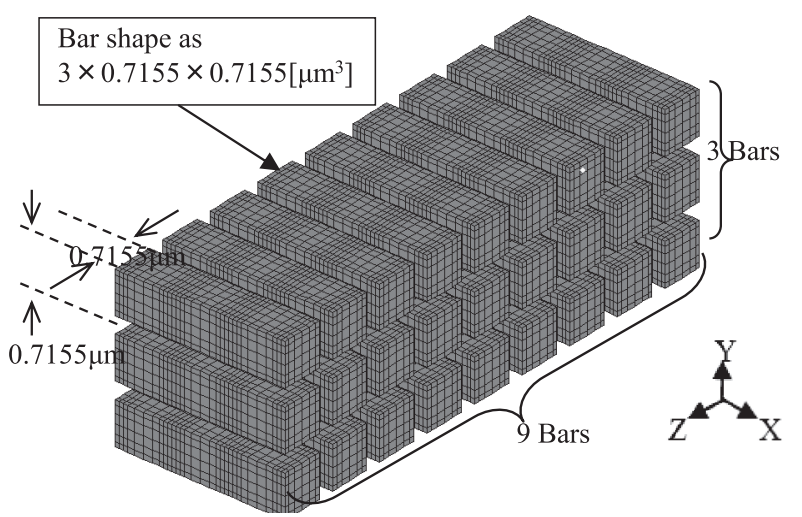

(a) Case-A

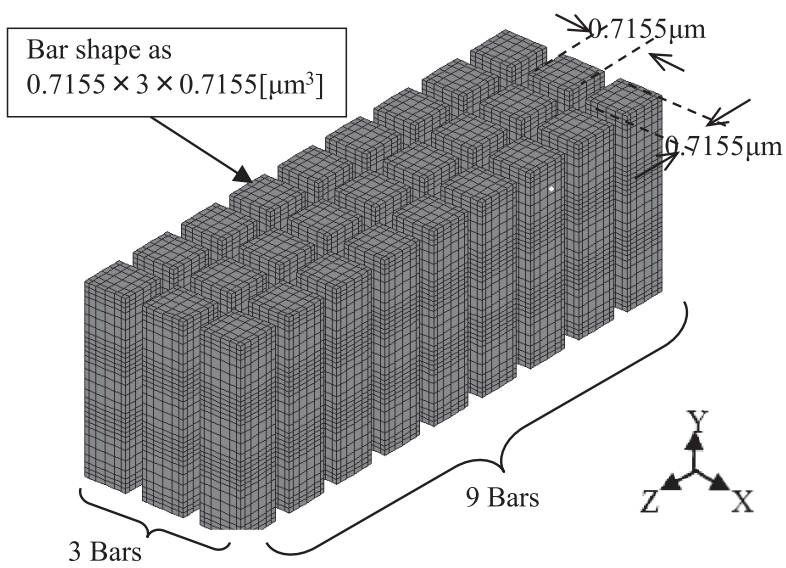

(b) Case-B

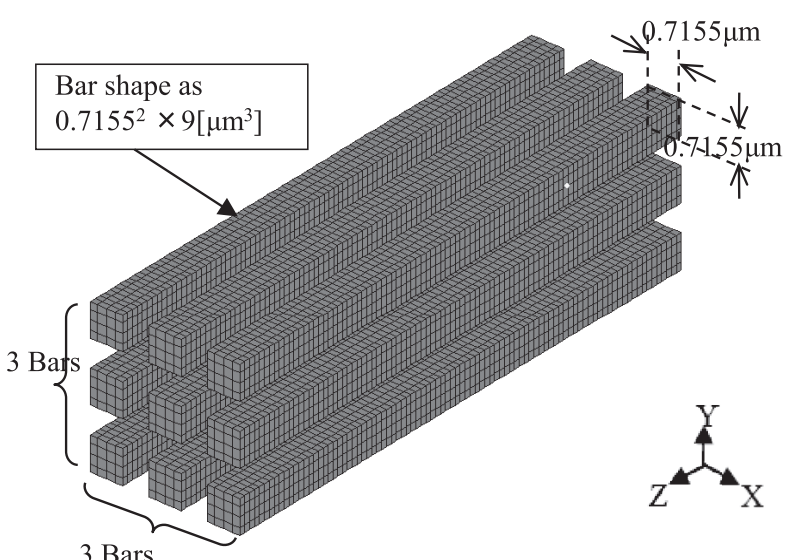

(c) Case-C

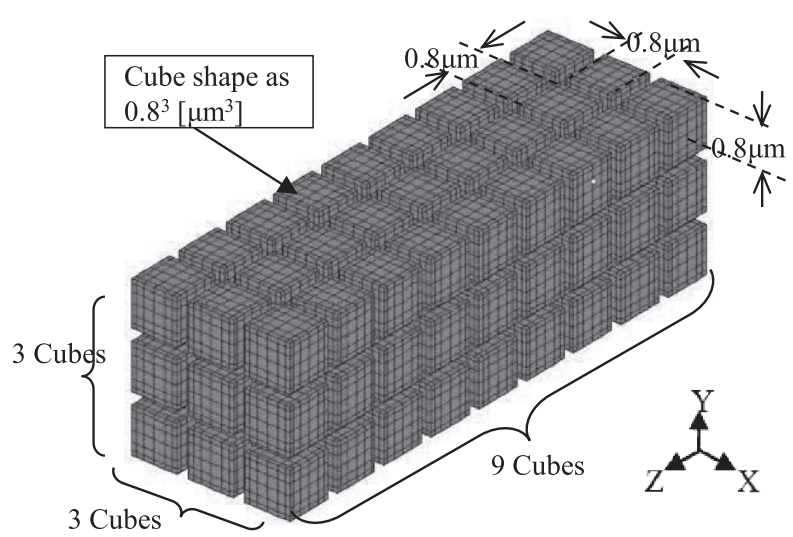

(d) Case-D

Fig. 4. Shape of dielectric material (water) in calculation condition, which is arranged in the center part of Figs. 2(a) 2(b), $2(\mathrm{c})$ are in shape of bars which has $0.7155^{2}\left[\mu \mathrm{m}^{2}\right]$ in cross section. (d) is 81 cubes. ison. Since the cube shape of micro-structure is symmetry in 3-dimensional space, the difference of the direction of applying electromagnetic field is not considered.

Case D is also arranged in the center part of Fig. 2. Then the numerical calculation for all the cases is done. Material constants are shown in Table $\mathbf{1 .}$

\section{Calculation Results and Discussion}

\subsection{Volume Averaged Electromagnetic Vector}

Through the numerical calculation, magnetic field and electrical field distribution, which are derived from vector potential and scholar potential in Eq. (1), is obtained.

The basic unit, which is $1 \mu \mathrm{m}^{3}$ cube in this model, is considered to be repeated endless in 3-dimensional space. Then a volume averaged electromagnetic vector is considered to be a physical vector of the bar shape structure from a macroview point by homogenizing the micro-structures. ${ }^{16-18)}$ Then the volume-averaged electromagnetic field per the basic unit is defined as follows: ${ }^{19)}$

$$
\dot{\vec{D}}_{a v e}=\frac{\sum \dot{\vec{D}}_{i} \Delta V_{i}}{\sum \Delta V_{i}}, \dot{\vec{E}}_{a v e}=\frac{\sum \dot{\vec{E}}_{i} \Delta V_{i}}{\sum \Delta V_{i}} .
$$

Since ferromagnetic material is not considered here, the volume averaged magnetic field and magnetic flux density is not much influenced by this calculation. The real part and the imaginary part of the derived relative magnetic permeability is almost 1 and 0 respectively. Therefore, only the electrical field is considered here.

Since electrical field is arranged only in Y-component as a boundary condition shown in Fig. 2, most of electrical field in micro-model is Y-component. Volume averaged operations as Eq. (2) is applied to Y-component only of electrical field. Table 2 shows the volume averaged electrical field and electrical flux density for case A-D. The averaged electrical field is almost the same for all cases, though the averaged electrical flux density is different. It means that the electrical flux density depends on the micro-structural arrangement of dielectric material in spite of the same volume rate.

Table 1. Material constants.

\begin{tabular}{lcccc}
\hline & $\begin{array}{c}\text { Relative magnetic } \\
\text { permeability }\end{array}$ & \multicolumn{2}{c}{$\begin{array}{c}\text { Relative dielectric } \\
\text { constant }\end{array}$} \\
& Real & Imaginary & Real & Imaginary \\
Unit & - & - & - & - \\
\hline Matrix: Air & 1 & 0 & 1 & 0 \\
Dielectric material: Water & 1 & 0 & 76.7 & 12.04 \\
\hline
\end{tabular}

Table 2. Calculation results.

\begin{tabular}{ccccc}
\hline & Case-A & Case-B & Case-C & Case-D \\
\hline$E_{y, \text { ave }}^{\text {real }}[\mathrm{V} / \mathrm{m}]$ & 1.00 & 1.00 & 1.00 & 1.00 \\
$E_{y, \text { ave }}^{\text {imag }}[\mathrm{V} / \mathrm{m}]$ & -0.00039 & -0.00039 & -0.00039 & -0.00039 \\
$D_{y, \text { ave }}^{\text {real }}\left[\mathrm{C} / \mathrm{m}^{2}\right]$ & $2.69 \mathrm{E}-11$ & $35.2 \mathrm{E}-11$ & $1.69 \mathrm{E}-11$ & $3.62 \mathrm{E}-11$ \\
$D_{y, \text { ave }}^{\text {imag }}\left[\mathrm{C} / \mathrm{m}^{2}\right]$ & $5.71 \mathrm{E}-12$ & $54.4 \mathrm{E}-12$ & $2.56-12$ & $8.49 \mathrm{E}-12$ \\
\hline
\end{tabular}




\subsection{Equivalent Material Constants and Shape Anisot- ropy}

The electrical field and electrical flux density defined in Eq. (2), which is volume-averaged per basic unit, is considered to be a macro-view physical value. It is useful to treat a macro-problem where micro-structure is too small to treat. Therefore, the equivalent relative dielectric constant $\left[\dot{\varepsilon}_{\text {ave }}\right]$ of the micro-structure is defined as follow: ${ }^{20)}$

$$
\dot{\vec{D}}_{\text {ave }}=\varepsilon_{0}\left[\dot{\varepsilon}_{\text {ave }}\right] \dot{\vec{E}}_{\text {ave }}
$$

Here, $\varepsilon_{0}$ is a vacuum dielectric constant. The relative dielectric constant should be considered as Y-component only, because of the direction of electrical field.

The equivalent relative dielectric constants in Eq. (3) are shown in Fig. 5 for case A-D. Since it is a complex number, real part and imaginary part are shown. For comparison, the value of "volume rate proportion", which multiplies the dielectric constant of Table 1 and the volume rate as $51.2 \%$, is also shown.

Though the case A-C treats the same object as Fig. 3 and only the applied directions are different, the relative dielectric constants are different each other. Namely it means that the micro-structure of bar shape has an anisotropy in electromagnetic field application.

The case $\mathrm{A}-\mathrm{C}$ corresponds to the conditions that the directions of electrical field are $\mathrm{y}-, \mathrm{z}-$ and $\mathrm{x}$-direction respectively in Fig. 3. Then the relative dielectric constant for case A-C can be regarded as the equivalent dielectric constant tensor ingredient for $\mathrm{y}-, \mathrm{z}-, \mathrm{x}-$ respectively. Here, the tensor is assumed to be a diagonal matrix. Then the equivalent relative dielectric constant tensor of the bar shape expresses as the next equation.

$$
\left[\dot{\varepsilon}_{a v e}\right]_{b a r}=\left[\begin{array}{ccc}
1.9+j 0.29 & 0 & 0 \\
0 & 3.0+j 0.65 & 0 \\
0 & 0 & 39.8+j 6.16
\end{array}\right] \ldots \ldots .
$$

Since each ingredient of the diagonal matrix in Eq. (4) is different, the bar shape is considered to have anisotropic char-

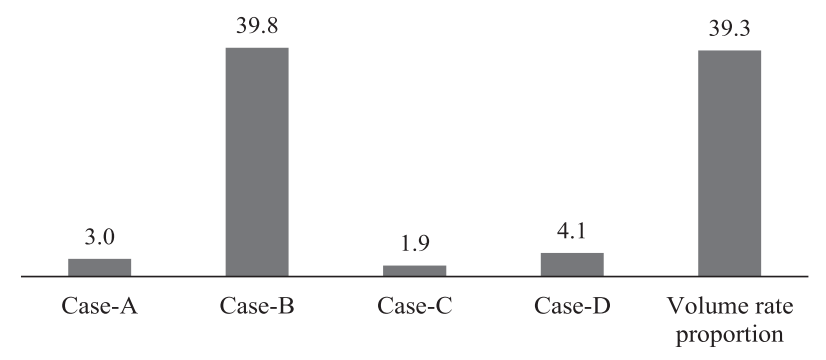

(a) Real part.

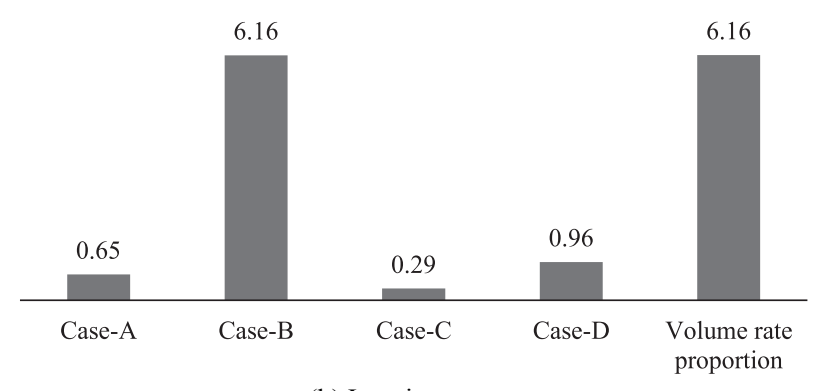

(b) Imaginary part.

Fig. 5. Equivalent relative dielectric constant defined as Eq. (3). acteristics of electromagnetic field.

Since the cube shape as case-D is symmetry in 3-dimensional space, the tensor of the cube shape becomes isotropy and expresses as the next equations.

$$
\left[\dot{\varepsilon}_{\text {ave }}\right]_{\text {cube }}=\left[\begin{array}{ccc}
4.1+j 0.96 & 0 & 0 \\
0 & 4.1+j 0.96 & 0 \\
0 & 0 & 4.1+j 0.96
\end{array}\right] \ldots .
$$

Therefore, bar shape micro-structure has anisotropic characteristics, though cube shape micro-structure has isotropic ones.

\subsection{Depolarization Field}

Though the equivalent relative dielectric constants are introduced in the same volume rate, Eqs. (4) and (5) show that they are different. The reason is considered as Fig. 5 in comparison with the volume rate.

The equivalent relative dielectric constant of the case-B is the largest among the case A-D and is almost the same value of the volume rate proportion. In the case- $\mathrm{B}$, the direction of the electrical field is the same as the one where the bar lengthens to infinity. In the other cases, the length of the bar is limited to the direction of the electric field. Since electric charges move to the direction of the electrical field, it can be considered to make them generate inside of the limited length of the bar. It is considered that the separated electrical charges generate a depolarization field inside it and make electrical flux density weaken as shown in Fig. 6. The generation of depolarization is considered to be the same phenomenon as the one of demagnetization in ferromagnetic body which magnetic field is applied to. ${ }^{21)}$

The equivalent relative dielectric constant becomes smaller than the volume rate proportion, when electromagnetic material is separated mechanically in electrical file application direction of unit size. The reason that the equivalent relative dielectric constant of case- $\mathrm{A}$ and $\mathrm{C}$ becomes smaller than that of case-D is considered to be that the dielectric material length in electrical field direction is smaller.

The experimental data of the permeability of $\mathrm{Fe}_{2} \mathrm{O}_{3}$ powder show this phenomenon; the permeability becomes under the volume rate of the permeability. ${ }^{15)} \mathrm{Fe}_{2} \mathrm{O}_{3}$ powder does not continue (separated) mechanically and air is inserted.

Therefore, the generation of depolarization field within the micro-structure causes the difference of electromagnetic characteristics and the anisotropy.

\section{Conclusion}

Electromagnetic anisotropic characteristics of electromagnetic materials in application of microwave are observed by micro-sized numerical high frequency electro-

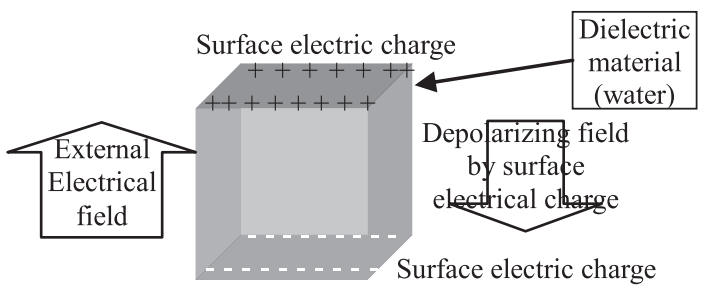

Fig. 6. Schematic view of depolarization field in dielectric material. 
magnetic computation. It is researched as the fundamental characteristics of microwave application, which is expected to be one of the useful technologies to solve $\mathrm{CO}_{2}$ problem in the reduction reaction of iron oxide. In assuming that the micro-sized unit structure is repeated in 3-dimensional directions and its size is much smaller than the electromagnetic wave length, the micro-sized electromagnetic field calculation of finite element method and $\mathrm{j} \omega$ method is carried out. In considering the bar shape and the cube shape as a micro-structure of electromagnetic material, the bar shape micro-structure has anisotropic characteristics, though cube shape micro-structure has isotropic ones. The generation of depolarization field within the micro-structure causes the difference of electromagnetic characteristics and the anisotropy.

\section{Acknowledgment}

Some part of this work was supported in part by Hi-tech Research Center Project for Private University from the Ministry of Education, Culture, Sports, Science and Technology. The author thanks for Dr. S. Satoh for his computational support.

\section{REFERENCES}

1) D. Adam: Nature, 421 (2003), 571.

2) M. Nuechter, U. Mueller, B. Ondruschka, A. Tied and W.
Lautenschlaeger: Chem. Eng. Technol., 26 (2003), 1207.

3) H. Takizawa, T. Kimura, M. Iwasaki, K. Uheda and T. Endo: Ceram. Trans., 133 (2002), 211.

4) N. Yoshikawa, H. Wang, K. Mashiko and S. Taniguchi: J. Mater. Res., 23 (2008), 1564.

5) M. Sato, A. Matsubara and K. Nagata: TMS Shon Symp.,TMS, Warrendale, PA, (2006).

6) H. Taira, Y. Saito, K. Uchinokura, K. Sawano, H. Kasahara, M. Amano, S. Sukenari and K. Kataoka: Taikabutu, 55 (2003), 19.

7) T. Kuroki, Y. Uchida, H. Takizawa and K. Morita: ISIJ Int., 47 (2007), 592.

8) K. Nagata and K. Ishizaki: ISIJ Int., 47 (2007), 811

9) S. Tae, T. Tanaka and K. Morita: ISIJ Int., 49 (2009), 1259.

10) R. Roy, D. Agrawal, J. Cheng and S. Gedevanishvili: Nature, 399 (1999), 668.

11) K. Fujisaki: INTERMAG2008, Digest of Technical Papers, IEEE, Piscataway, NJ, USA, (2008), CV-06.

12) M. Suzuki, M. Ignatenko, M. Yamashiro, M. Tanaka and M. Sato: ISIJ Int., 48 (2008), 681

13) K. Fujisaki: ISIJ Int., 49 (2009), 1636.

14) K. Watanabe, S. Ueda, R. Inoue and T. Ariyama: ISIJ Int., 50 (2010), 524.

15) M. Hotta, M Hayashi and K. Nagata: ISIJ Int., 50 (2010), 1514.

16) K. Fujisaki, M. Fujikura, J. Mino and S. Satou: IEEE Trans. Magn., 46 (2010), 1147.

17) S. Odawara, Y. Haraguchi, K. Muramatsu, K. Yamazaki and S. Hirosato: IEEE Trans. Magn., 46 (2010), 3313.

18) H. Kaimori, A. Kameari and K. Fujiwara: IEEE Trans. Magn., 43 (2007), 1405.

19) H. Waki, H. Igarashi and T. Honma: IEEE Trans. Magn., 42 (2006), 847.

20) K. Fujisaki and S. Satou: IEEE Trans. Magn., 44 (2008), 3161.

21) S. Chikazumi: Physics of Magnetism, John Wiley \& Sons, New York, (1964). 